\title{
Percepção de estudantes de graduação em Odontologia frente ao atendimento de pessoas com deficiência
}

\author{
Simone Helena Ferreira*; Raiza Alves Suita**; Priscila Humbert Rodrigues***; Paulo Floriani \\ Kramer***
}

* Mestre, professora na Universidade Luterana do Brasil/Canoas, Coordenadora do Projeto de Extensão "Conquistando Saúde:

Atendimento Odontológico de Pessoas com Deficiência”

** Cirurgiã-dentista graduada pela Universidade Luterana do Brasil/Canoas

*** Doutor, Professor na Universidade Luterana do Brasil/Canoas

Recebido em 27/12/2016. Aprovado em 25/01/2017.

\begin{abstract}
RESUMO
O objetivo deste estudo foi identificar e analisar a percepção de estudantes frente ao atendimento de pessoas com deficiência no projeto de extensão "Conquistando saúde: atendimento odontológico de pessoas com deficiência" da Universidade Luterana do Brasil (ULBRA), em Canoas/RS. O delineamento do estudo foi qualitativo e envolveu 13 estudantes. Os dados foram obtidos por meio de entrevista semiestruturada realizada no ambulatório do curso de Odontologia por um pesquisador treinado e os dados foram analisados por análise de conteúdo. As questões versavam a respeito do conhecimento em relação a pessoas com deficiência, a motivação para participar do projeto e os sentimentos norteadores dos atendimentos. A maioria dos entrevistados modificou opiniões existentes sobre o que acreditavam ser uma pessoa com deficiência e seus sentimentos prévios aos atendimentos, acrescentando novos conceitos e sentimentos que fomentaram sua construção profissional. A oportunidade de vivenciar o atendimento odontológico de pessoas com deficiências ainda na graduação em Odontologia contribui para a formação de um profissional mais capacitado tanto técnica quanto emocionalmente. Com isto, há ampliação do atendimento, minimizando a dificuldade enfrentada por estes pacientes na busca pelo atendimento odontológico.
\end{abstract}

Descritores: Odontologia para Pessoas com Deficiência. Percepção. Desenvolvimento de Pessoal.

\section{INTRODUÇÃO}

A convenção sobre os "Direitos das Pessoas com Deficiência" destaca que pessoas com deficiência (PcD) são aquelas que apresentam restrições de natureza física, intelectual ou sensorial que impossibilitam sua participação integral e permanente na sociedade ${ }^{1}$. Mais de um bilhão de pessoas em todo o mundo convivem com alguma forma de deficiência, dentre estas $20 \%$ possuem dificuldades 
funcionais consideráveis. No Brasil, aproximadamente $1 / 4$ da população tem algum tipo de deficiência ${ }^{2,3}$.

Disfunções sistêmicas em PcD predispõem a doenças na cavidade bucal, as quais, por sua vez, podem agravar doenças sistêmicas. Assim, indivíduos com deficiência necessitam de atenção médica e odontológica voltadas especificamente à sua condição ${ }^{4}$; e os cirurgiões dentistas devem estar preparados para oferecer tratamento adequado que contemple as necessidades diagnosticadas 5,6 .

Estudos têm demonstrado que PcD encontram dificuldades no acesso ao atendimento odontológico ${ }^{7}$ e os profissionais relatam desde eventuais dificuldades técnicas até o envolvimento emocional que modula este tipo de atendimento ${ }^{8}$.

De acordo com as Diretrizes Curriculares Nacionais (DCN) dos Cursos de Graduação em Odontologia, o cirurgião-dentista deve ter uma formação generalista, humanista, crítica e reflexiva, pautada em princípios éticos, legais e na compreensão da realidade socioeconômica e cultural do seu meio. Neste sentido, é essencial que a sua formação o qualifique a lidar com a diversidade de forma técnica e humana. O conhecimento especializado é necessário e pode ser adquirido por formação complementar, aumentando assim a consciência e a atenção, indispensáveis no atendimento clínico de pacientes com deficiência ${ }^{9-12}$.

Cirurgiões-dentistas que pretendem dedicar-se ao atendimento de PcD devem tratar a todos com dignidade, respeito e cuidado ético, independente da deficiência. A preparação destes profissionais requer, além de formação técnica, sensibilização e predisposição ao atendimento, resultando na atenção odontológica com maior qualidade $^{1,2,8,13}$.

Desde 2002 a especialidade "Odontologia para Pacientes com Necessidades Especiais” é regulamentada pelo Conselho Federal de Odontologia (CFO) com o objetivo de capacitar os cirurgiões-dentistas ao atendimento de pessoas que necessitam de cuidados odontológicos especiais durante toda a vida ou por um período. O CFO utiliza-se do termo "Pacientes com Necessidades Especiais" por considerar que limitações temporárias ou permanentes, de ordem intelectual, física, sensorial, emocional, de crescimento ou condições sistêmicas crônicas impedem um atendimento odontológico de rotina ${ }^{2,14}$.

Estudantes dos cursos de graduação, especialmente após a implantação de políticas inclusivas, podem já ter tido experiências com estes indivíduos. Por isso, sua forma de pensar e sentir, no que se refere a este tipo de atendimento, deve ser analisada para que possíveis mudanças na sua formação possam ser introduzidas ${ }^{1,3,11}$.

O curso de graduação em Odontologia da ULBRA/Canoas oferece aos seus estudantes a participação no Projeto de Extensão "Conquistando Saúde: Atendimento Odontológico de Pessoas com Deficiência", como atividade voluntária para acadêmicos do último ano do curso. Este projeto, além de viabilizar o atendimento odontológico destes cidadãos, oportuniza o crescimento profissional dos acadêmicos. Os pacientes atendidos vêm da região metropolitana de Canoas e de outros municípios do Rio Grande do $\mathrm{Sul}^{15}$.

O presente estudo teve como objetivo identificar e analisar, de forma qualitativa, a percepção dos estudantes voluntários do Projeto de Extensão "Conquistando Saúde: Atendimento Odontológico de Pessoas com Deficiência" frente a estes atendimentos.

\section{METODOLOGIA}

O delineamento do estudo é qualitativo por meio de entrevistas. Neste tipo de estudo os resultados produzidos procedem da percepção, 
conhecimentos e emoções de cada sujeito envolvido a cerca do tema a ser estudado. A abordagem qualitativa procura aprofundar o conhecimento de forma dinâmica e flexível ${ }^{16}$. O presente estudo seguiu os critérios do Consolidated criteria for reporting qualitative research (COREQ) ${ }^{17}$.

O tamanho da amostra não é fator determinante de significância no estudo qualitativo, que trabalha com amostras relativamente pequenas de forma intencional e selecionada ${ }^{18}$. Deste modo, os 13 acadêmicos voluntários que participaram do projeto de extensão no primeiro semestre de 2015 foram o público alvo desta pesquisa e o processo de investigação foi realizado no Curso de Odontologia da ULBRA Canoas/RS.

Os estudantes responderam a uma entrevista composta por questões norteadoras que versavam a respeito do conhecimento em relação a $\mathrm{PcD}$, a motivação como estudante para participar do projeto, bem como os sentimentos que nortearam os atendimentos. Os entrevistados foram identificados por numeração.

A coleta de dados foi realizada por um pesquisador treinado, por meio de entrevista semiestruturada gravada. As entrevistas foram transcritas integral e literalmente pelo entrevistador. A seguir, foi realizado o processo de análise de conteúdo proposto por Bardin $(1977)^{18}$, no qual as respostas são categorizadas e analisadas.

O termo "análise de conteúdo" não significa somente um procedimento técnico, mas também busca teoria e prática no campo das investigações sociais, sendo definido como "um conjunto de técnicas de análise de comunicação visando obter, por procedimentos sistemáticos e objetivos de descrição de conteúdo das mensagens, indicadores que permitam a inferência de conhecimentos relativos às condições de produção/recepção destas mensagens" $" 18$.

O projeto foi submetido ao Comitê de Ética em Pesquisa da ULBRA e aprovado sob parecer 922.030. As entrevistas iniciaram após a autorização do entrevistado por meio do Termo de Consentimento Livre e Esclarecido.

\section{RESULTADOS E DISCUSSÃO}

Os resultados apresentados correspondem a recortes das falas transcritas pelo pesquisador obtidas por análise de conteúdo. Esta análise originou as grandes categorias que serão apresentadas e discutidas a seguir.

Com relação à definição do que seja $\mathrm{PcD}$ alguns entrevistados mostraram indecisão nas respostas, o que pode ser evidenciado nestes relatos: "Que tenha alguma dificuldade, algum[...] Como que eu posso dizer? Alguma limitação ou em movimento ou mental, eu acho que é isto" (E6); "Não sei!..." (E10); "Como posso te dizer isso?...” (E12). Esta dificuldade em definir o que é deficiência justifica-se pela falta de contato com estes pacientes durante o Curso de Graduação quando comparado a outros atendimentos clínicos presentes na grade curricular do curso.

Coyle et al. (2004) $)^{19}$, avaliando atitudes de estudantes de Odontologia e de ciências sociais relacionadas ao ensino sobre pessoas com necessidades especiais, perceberam que os estudantes de Odontologia parecem ter menos facilidade de aprender sobre estas pessoas em comparação com os estudantes de ciências sociais. Os autores destacam a falta de políticas e práticas educacionais na formação profissional em saúde. Por outro lado, Moraes et al., (2006) ${ }^{11}$, analisando as falas de estudantes de Odontologia sobre PcD perceberam que estes estão predispostos a ações inclusivas, desde que recebam informações sobre isto.

Ainda com relação ao conceito de $\mathrm{PcD}$ alguns relatos assemelham-se: “...Alguma 
alteração neurológica, ou impossibilidade de movimentos, acho que seria isto.” (E2), “... total falta de independência” (E3), “...Deficiência física[...] Que não tem condições mentais para entender como se deve proceder, como se faz a higiene [...] Precisa de monitoramento 24 horas" (E4). Nestas respostas é possível verificar que os estudantes associam a deficiência com incapacidade de viverem sozinhos, necessitando de cuidados permanentes. Esta percepção vai ao encontro da definição de deficiência do Decreto $\mathrm{n}^{\circ} 1.744$ de 1995 que diz: "Pessoa com deficiência é aquela que apresenta incapacidade para a vida independente e para o trabalho em razão de anomalias ou lesões irreversíveis de natureza hereditária, congênita ou adquirida, estando, portanto, impedida de desempenhar as atividades de vida diária e de trabalhar" ${ }^{20}$.

De acordo com esta definição podemos perceber a importância do papel dos pais ou responsáveis na vida destes indivíduos. É comum que a procura por um tratamento odontológico seja demorada em função de prioridades de atendimento médico. Além disso, é possível que o baixo nível cultural e socioeconômico faça com que esta demora seja algo habitual. Por outro lado, verifica-se que o tratamento odontológico é uma das maiores necessidades para essa população $^{14,21}$.

Ainda com relação à definição, um entrevistado descreve: "Uma pessoa que precisa de uma atenção especial, que precisa de um cuidado diferenciado e que tem que ser tratada de um jeito diferente, mas, ao mesmo tempo com respeito e[...] respeitando as particularidades de cada um. Que todo mundo é diferente, né?" (E13). O entendimento do mundo particular de cada indivíduo é vital para a compreensão do outro. Seja qual for a percepção do profissional ao atender estes pacientes ele deve alforriar-se de preconceitos e tabus ${ }^{22}$.
Quando os estudantes foram indagados sobre a motivação para participarem do projeto, grande parte relatou sentir curiosidade e vontade de adquirir novos conhecimentos, esboçando interesses semelhantes, como vistos nestes trechos: "... pra saber como que a gente atenderia este tipo de pessoas..." (E1), "Porque eu acho importante a gente saber lidar com este tipo de paciente..." (E3), "Fiquei interessada em conhecer melhor. Para as minhas habilidades" (E9).

A preocupação com o futuro profissional e a oportunidade de ter contato com $\mathrm{PcD}$, ainda na graduação, também foram motivos para justificar a procura pelo projeto: “... pode ser que venha na nossa clínica, no nosso futuro que a gente não vai saber como lidar [...] não saber como ter um jeito de conversar, de posicionar na cadeira..." (E3), “... E porque quando a gente sair daqui, a gente nunca sabe o que vai fazer...” (E4), “... Coisa que a gente não tem nas clínicas normais né?” (E5), “... eu acredito que isto vai agregar muito para nossa formação, até porque durante as nossas clínicas normais a gente não tem contato..." (E11), "Pra vivenciar uma coisa que não ia vivenciar se eu não fizesse o projeto" (E13).

As dificuldades associadas ao tratamento odontológico para PcD iniciam-se pela ausência da disciplina de Odontologia para Pacientes com Necessidades Especiais na graduação na maioria das Faculdades de Odontologia do Brasil ${ }^{23}$. Cirurgiões-dentistas sem esta vivência na universidade criam uma barreira no momento do atendimento destes indivíduos. Desta forma, podemos destacar a importância destas experiências na construção do futuro profissional. O conhecimento técnico associado à compreensão dos fatores relacionados à pacientes com deficiências permite uma inserção mais madura no mercado de trabalho, além de 
desencadear mudanças em valores e disposições afetivas. $11,14,17,22,24$

Outro estudante entrevistado elucida de forma simples o que o motivou a participar do projeto: “... principalmente por medo de quando eu sair daqui de não saber atender essas pessoas, eu acho que aqui dentro da faculdade é a melhor oportunidade que a gente tem de aprender, porque a gente tem professores que podem nos auxiliar melhor. Lá fora eu sozinha talvez eu não saiba tanto lidar com isso, né? Então, é bom já sair daqui pelo menos com uma base disto" (E6).

Dos 13 entrevistados, 12 relataram que nunca haviam tido experiência com PcDs. O estudante que já havia tido experiência, esta havia sido no próprio projeto: "É a segunda vez que eu estou participando do projeto, então, pra mim tá sendo muito mais legal assim [...] Dá para entender certas coisas já, eu também acho que to ajudando outros colegas que estão fazendo pela primeira vez [...] Eu acho que toda vez tem uma surpresa neste sentido para a gente aprender" (E7). Analisando este relato é possível observar que esta vivência auxilia na capacitação dos estudantes e também faz com que eles se tornem mais colaborativos e tenham uma maior resolutividade no atendimento dentro e fora do âmbito universitário ${ }^{12}$.

Algumas escolas de Odontologia têm criado serviços especializados no atendimento de $\mathrm{PcD}$, o que pode, de certa forma, ampliar as ações de segregação. De acordo com a perspectiva inclusiva, seria importante buscar estratégias de atendimento junto aos pacientes sem deficiência ${ }^{11}$. Entretanto, frente as respostas do presente estudo, verifica-se a disposição favorável dos estudantes em atender pessoas com deficiência e observar que as dificuldades podem ser superadas no processo de atuação. $\mathrm{O}$ estudante passa a ser um agente promotor de ações de inclusão ${ }^{11}$.
A ideia de fazerem do atendimento odontológico à PcD uma prática clínica fora da universidade se mostrou presente em praticamente todos os relatos: "Atenderia sim [...] Com mais alguém auxiliando, ajudando pra evitar possíveis acidentes né?..." (E3), "Trabalharia, mas não trabalharia sozinha, né??” (E4), “...de repente tu não consiga atender alguns, porque tu precisa de mais pessoas, precisa de mais pessoas disponíveis pra te ajudarem né?" (E5). Torna-se claro que a condição para tal seria a presença de pessoal auxiliar, já que a vivência dentro da universidade comprova que o trabalho em equipe nestas intervenções é essencial.

Apenas um estudante entrevistado não demonstrou interesse em trabalhar com PcD após formado alegando não estar preparado psicologicamente para este tipo de atendimento. Isto demonstra que nem todos os estudantes têm perfil para isto. Em estudo realizado para avaliar os sentimentos e expectativas de acadêmicos do Curso de Odontologia frente ao atendimento de pessoas com necessidades especiais, $54 \%$ deles entendem que atender esses pacientes é uma vocação e $70 \%$ deles responderam não ter certeza que atenderiam PcD ou nunca fariam essa especialidade ${ }^{25}$.

Entretanto, a vivência dos estudantes na graduação contribui na formação de valores, diminuição dos sentimentos negativos e construção de um cirurgião dentista mais completo $^{11}$. Isto pode ser observado neste trecho: "Eu tive essa mudança de percepção, assim, no atendimento não só para eles, mas, com os pacientes em geral" (E8). As emoções dos estudantes devem ser compartilhadas, pois a elaboração de sentimentos orienta atitudes, ações e reações, levando a um ganho maior de autoconfiança durante $\mathrm{o}$ atendimento desses pacientes $^{25}$. 
A formação de um cirurgião dentista capacitado tecnicamente e que cultive valores que acolham também a família podem minimizar as dificuldades encontradas no acesso ao atendimento odontológico 22,21 . Podemos perceber isto nesta fala: "Além de poder ajudar essas pessoas que às vezes não têm atendimento por profissionais que tem medo, eu gostaria de ajudar elas de alguma forma, como profissional e também às vezes dando uma orientação que essa família não tem. Eu acho que é fazer além da parte clínica, mas, a parte humana do tratamento" (E7).

No decorrer da entrevista os estudantes foram questionados sobre a impressão que tiveram do primeiro atendimento realizado a PcD. Grande parte respondeu que sentiu medo e pena dos pacientes e seus familiares: "Fiquei com muita pena, muita, muita, muita, porque a gente vê a situação deles né?..." (E2), "Na primeira vez eu estava um pouquinho nervosa, um pouquinho com medo..." (E8), "Acho que uma mistura de sentimentos, emoções, é medo, é pena, até se colocar igual elas né?...” (E9). Estes sentimentos ressaltam o envolvimento emocional durante o atendimento. Além disto, os atendimentos se tornam mais demorados devido à dificuldade de cooperação dos pacientes ${ }^{8}$.

Os medos, inseguranças e dificuldades expostas pelos entrevistados devem ser levados em conta como uma situação a ser superada. As respostas que expressam, inicialmente, medo e receio diminuem com o tempo e são seguidas pela busca de informações, conhecimento e predisposição ao atendimento. A adaptação adequada dos estudantes por meio do conhecimento facilita a realização dos procedimentos $^{8,11,13}$.

No estudo de Amaral et al. (2011) ${ }^{25}$, realizado para avaliar os sentimentos e expectativas de acadêmicos do Curso de Odontologia frente ao atendimento de $\mathrm{PcD}$ percebem-se sentimentos ambivalentes. Os sentimentos negativos mais comuns foram insegurança, medo, tristeza, além da dificuldade em atender o paciente; e os positivos, envolveram a necessidade de cuidar com amor, carinho e responsabilidade.

A forma de abordar os pacientes também foi uma preocupação constante dos entrevistados frente ao primeiro atendimento. Por outro lado, em quase todos os relatos os estudantes expuseram a ideia de que com o decorrer dos atendimentos e a prática clínica o aprendizado ia se aprimorando, como visto neste trecho: “...O que eu vou fazer? Como que eu vou falar? Como é que eu vou agir com a mãe? Com ele... Às vezes em certas situações que acontecem a gente não sabe como fazer; mas eu acho que a gente vai aprendendo com a própria mãe às vezes, e com outras situações que acontecem ali na clínica." (E7).

Mudanças na percepção dos estudantes em relação à $\mathrm{PcD}$, a partir do convívio semanal puderam ser percebidas: “...antes queria digamos, fugir, entendeu? Não sabia como ia ser, o que ia acontecer e agora já não..." (E1), "Antes eu senti um pouco de pena, mas, eu fui entender assim, que né? ...eu acho que é ajudar e não sentir pena” (E7). “...Eu acho que me fez ver [...] tem muito preconceito, tem muito essa coisa de discriminação, né? $E$, no fundo não precisa ter isso, né? ... E eu acho legal o projeto que tá fazendo a gente, além de conhecer toda essa função de trabalho e tudo mais, vai ter esse diferencial lá fora, e em relação a eles... Eles são, acho que são mais do que especiais, né?" (E5). Percebe-se também nas falas que a evolução era evidente a cada atendimento: “...eu acho que já "abriu bastante à cabeça” e eu vou saber lidar pelo menos acho que uns $70 \%$ mais do que antes eu não saberia[...] Não dando para apropriar a técnica correta, a gente tentando outro manejo para poder anestesiar o paciente 
ou escovar de outra maneira para ajudar eles, né? Eu acho que vou saber lidar com isso bem mais daqui pra frente[...] Que dirá no fim do semestre tendo mais experiência, né? Eu acho que tá sendo muito bom." (E3). Estas mudanças demonstram que a vivência contribui para a construção de um profissional consciente e humano; e que experiências clínicas motivadoras geram destreza e autoconfiança nos estudantes, ${ }^{22}$.

Com relação ao conhecimento específico da área da Odontologia, os acadêmicos comentaram que a técnica odontológica a ser utilizada nos procedimentos é a mesma, entretanto, a forma de abordar e lidar com o paciente é que é diferente: " $E$, que acho que a técnica ali ela é a mesma, mas, ela precisa ter agilidade né? ...” (E4), “...Só que eu acho que como abordar [...] a abordagem eu acho que muda né? ..." (E2), "É que o que muda é como tu vai lidar com eles né?..." (E9).

Além do aprendizado clínico, saber lidar com as emoções para que o atendimento seja resolutivo é fundamental. $\mathrm{O}$ próprio envolvimento pessoal também fez parte da fala deste entrevistado: "Sabe que eu me senti, assim [...] Na verdade, muito emocionada, sabe? Até no segundo atendimento assim eu me pisquei, porque eles têm uma história de vida sabe?... Um percurso sabe? Que é muito emocionante, né? Eu acho que a gente se apega muito, né? Olha meu olho! [...] Eu adorei! Eu não pensava que eu ia gostar tanto quanto eu estou gostando." (E5)

Ao serem indagados sobre o sentimento que tiveram ao término de cada atendimento os estudantes relataram opiniões semelhantes: “...Eles têm um amor muito grande, um carinho muito grande pela gente. Também, são muito agradecidos pelo o que a gente faz..." (E2), "Senti em parte um alivio de poder ter ajudado a pessoa de alguma forma, né?" (E3), "Missão cumprida, aquela coisa de[...] Consegui!" (E9). “...Então, é a forma diferente que tem de agradecer, é um olhar né?[...] A gente satisfeito por ter feito o trabalho da gente, e eles saem dali satisfeitos também, né?" (E5), "Eu fico satisfeita em[...] com o trabalho e de poder ajudar, e fico valorizando a minha vida, né?" (E12).

Os sentimentos de gratidão, dever cumprido e até mesmo a valorização da vida estavam presentes em grande parte dos relatos. No estudo qualitativo de Keselyak et al. (2007) ${ }^{26}$, estes sentimentos também foram relatados, além da conscientização das questões sociais relacionadas neste tipo de atendimento.

Alguns estudantes demonstraram o interesse em fazer especialização em Odontologia para Pacientes com Necessidades Especiais, tendo como meta aumentar o seu conhecimento na área e proporcionar um atendimento especializado fora da universidade. Importante salientar que a especialidade promove atenção a estes pacientes evoluindo de uma preocupação de caráter beneficente para uma concepção mais profissional ${ }^{11}$.

\section{CONCLUSÃO}

De acordo com as respostas obtidas através das entrevistas realizadas com acadêmicos voluntários no Projeto de Extensão: "Conquistando Saúde: Atendimento Odontológico de Pessoas com Deficiência" foi possível perceber mudança de conceitos préestabelecidos em entendimento no que se diz respeito a estes indivíduos; transformação de sentimentos iniciais de medo, insegurança e pena em vontade de entender e adaptar-se aos limites impostos por estes atendimentos; intenção de buscar mais conhecimento nesta área da Odontologia; conscientização de que a oportunidade desta experiência durante a graduação auxilia a construção de um profissional técnico e humanamente mais preparado fora do âmbito universitário, além de minimizar a dificuldade enfrentada por estes 
pacientes na busca pelo atendimento odontológico.

Considerando-se que grande parte dos cirurgiões-dentistas não está preparada para o atendimento odontológico de $\mathrm{PcD}$ e que estes encontram dificuldades de encontrar serviços odontológicos apropriados, acredita-se que conhecer melhor os sentimentos, percepções e manifestações dos futuros profissionais poderá auxiliar e fundamentar as reformas curriculares com o objetivo de beneficiar a qualificação profissional e a saúde bucal das pessoas envolvidas.

\section{ABSTRACT \\ Perception of Dental undergraduate students related to the attendance of special needs people or people with disabilities.}

This study aimed to identify and to analyze students' perceptions regarding the assistance of people with disabilities in the extension project "Conquering health: dental assistance of people with disabilities", of Universidade Luterana do Brasil (ULBRA) in Canoas/RS. It is a qualitative study developed with 13 students. Data were collected by a trained researcher using semistructured interviews in the Dental College Clinic and were analyzed with content analysis. The questions approached participants' knowledge regarding people with disabilities, their motivations to take part of the project, and feelings guiding their assistance. Most participants changed their opinions about what they believed to be a person with disabilities as well as their previous feelings towards assistance, adding new concepts and feelings that encourage professional performance. The opportunity to deeply experience dental assistance of people with disabilities while attending undergrad dentistry school contributes to the development of better professionals, both technically and emotionally. Therefore, there is an amplification of dental assistance, minimizing difficulties faced by patients with disabilities when looking for dental assistance.

Descriptors: Dental Care for Disabled. Perception. Staff Development.

\section{REFERÊNCIAS}

1. Resende AC, Vital FMP Organizadores. A Convenção sobre Direitos das Pessoas com Deficiência comentada. Brasília: Secretaria Especial dos Direitos Humanos, 2008.

2. Junior AFC, Machiavelli JL. Atenção e cuidado da saúde bucal da pessoa com deficiência. Introdução ao estudo. 1 ed. Recife: Editora Universitária da UFPE; 2013.

3. World Health Organization. World Report on Disability 2011. (Acesso em: 15 jun. 2015). Disponível em: http://www.who. int/disabilities/world_report/2011/report.pdf

4. Akhter R, Hassan NM, Martin EF, Muhit M, Haque MR, Smithers-Sheedy $\mathrm{H}$ et al. Risk factors for dental caries among children with cerebral palsy in a low-resource setting. Dev Med Child Neurol. 2016 Nov: 1-6. DOI:10.1111/dmcn.13359.

5. Resende VLS, Castilho LS, Souza ECV, Jorge WV. Atendimento odontológico a pacientes com necessidades especiais. In: $8^{\circ}$ Encontro de Extensão da UFMG; 2005 p.1 $-6$.

6. Vellappally S, Gardens SJ, Al Kheraif AA, Krishna M, Babu S, Hashem M, Jacob V, The prevalence of malocclusion and its association with dental caries among 12-18year-old disabled adolescents. Anil S BMC Oral Health. 2014;14:123. DOI: 10.1186/14 72-6831-14-123.

7. Shyama M, Al-Mutawa SA, Honkala E, Honkala S. Parental perceptions of dental visits and access to dental care among disabled schoolchildren in Kuwait. 
Odontostomatol Trop. 2015; 38(149):34-42.

8. Gerreth K, Lewicka MB. Access barriers to dental health care in children with disability. A questionnaire study of parents. J Appl Res Intellect Disabil. 2016;29(2): 139-45.

9. American Academy of Pediatric Dentistry. Definition of special health care needs. Pediatr Dent. 2012;36(6):16.

10. Conselho Nacional de Educação Câmara de Educação Superior. Resolução CNE/CES 3 de 19 de fevereiro de 2002. (Acesso em: 15 jun. 2015). Disponível em: http://portal. mec.gov.br/cne/arquivos/pdf/CES032002.pdf

11. Moraes ABA, Batista CG, Lombardo I, Horino LE, Rolim GS. Verbalizações de alunos de odontologia sobre a inclusão social de pessoas com deficiência. Psicol Estud. 2006;11(3): 607-15.

12. Mota LQ, Farias DBLM, Santos TA. Humanização no atendimento odontológico: acolhimento da subjetividade dos pacientes atendidos por alunos de graduação em Odontologia. Arq Odontol. 2012; 48(3):151-8.

13. Casamassimo PS, Seale NS, Ruehs K. General dentist's perceptions of educational and treatment issues affecting access to care for children with special health care needs. J Dent Educ. 2004; 68(1):23-8.

14. Fonseca ALA, Azzalis LA, Fonseca FLA, Botazzo C. Análise qualitativa das percepções de cirurgiões dentistas envolvidos nos atendimentos de pacientes com necessidades especiais de serviços públicos municipais. Rev Bras Crescimento Desenvolv Hum. 2010; 20(2):208-16.

15. Pereira LM, Mardero E, Ferreira SH, Kramer PF, Cogo RB. Atenção odontológica em pacientes com deficiência: a experiência do curso de Odontologia da ULBRA Canoas/RS. Stomatos 2010; 16(31):92-9.
16. Nico LS, Bocchi SCM, Ruiz T, Moreira RS. A grounded theory como abordagem metodológica para pesquisas qualitativas em odontologia. Ciênc Saúde Coletiva 2007;12(3):789-97.

17. Tong A, Sainsbury P, Craig J. Consolidated criteria for reporting qualitative research (COREQ): a 32-item checklist for interviews and focus groups. Int $\mathrm{J}$ qual Health Care. 2007; 19(6):349-57.

18. Bardin L. Análise de conteúdo. Lisboa: Edições 70; 1977.

19. Coyle C, Saunderson W, Freeman R. Dental students, social policy students and learning disabilities: do differing attitude exist? Eur J Dent Educ. 2004;8(3):133-9.

20. Brasil. Decreto ${ }^{\circ} 1.744$, de 8 de Dezembro de 1995.Regulamenta a Lei $n^{\circ} 8.742$, de 7 de dezembro de 1993, que estabelece o benefício de prestação continuada devido à pessoa portadora de deficiência e ao idoso e dá outras providências. Diário Oficial da República Federativa do Brasil, Poder Executivo, Brasília, DF, 11, dez. 1995. (Acesso em: 15 jun. 2015). Disponível em: http://www2.camara.leg.br/legin/fed/decret/ 1995/decreto-1744-8-dezembro-1995431802-norma-pe.html

21. Oliveira ALBM, Girro EMA. Importância da abordagem precoce no tratamento odontológico de pacientes com necessidades especiais. Odonto 2011; 19(38): 4551.

22. Cancino CMH, Oliveira FAM, Engers ME, Weber JBB, Oliveira MG. Odontologia para pacientes com necessidades especiais percepções, sentimentos e manifestações de alunos e familiares de pacientes. [Tese de Doutorado]. Porto Alegre: Faculdade de Odontologia - Pontifícia Universidade Católica do Rio Grande do Sul; 2005. 23. Haddad AS, Tagle EL, Passos VAB. 
Momento atual da Odontologia para pessoas com deficiência na América Latina: situação do Chile e Brasil. Rev Assoc Paul Cir Dent. 2016;70(2):132-40.

24. Dehaitem MJ, Ridley K, Kerschbaum WE, Inglehart MR. Dental hygiene education about patients with special needs: a survey of U.S. programs. J Dent Educ. 2008;72(9):1010-9.

25. Amaral COF, Aquotte APC, Aquotte LC, Parizi AGS, Oliveira A. Avaliação das expectativas e sentimentos de alunos de odontologia frente ao atendimento de pacientes com necessidades especiais. RFO UPF. 2011;16(2):124-9.
26. Keselyak NT, Simmer-Beck M, Bray KK, Gadbury-Amyot CC. Evaluation of an academic service-learning course on special needs patients for dental hygiene students: a qualitative study. J Dent Educ. 2007;71(3) :378-92.

Correspondência para:

Prof. Dr. Paulo Floriani Kramer e-mail: paulokramer@ hotmail.com

Curso de Odontologia - ULBRA

Av. Farroupilha, 8001

92425-900 Canoas/RS, 